

free content
- Additional supplementary files are published online only. To view these files please visit the journal online (http://dx.doi org/10.1136/emermed-2011201011).

${ }^{1}$ Accident and Emergency Medicine Academic Unit, The Chinese University of Hong Kong, Prince of Wales Hospital Shatin, Hong Kong SAR ${ }^{2}$ Accident and Emergency Department, Prince of Wales Hospital, Shatin, Hong Kong SAR

\section{Correspondence to} Josephine Yuen Man Chung, Accident and Emergency Medicine Academic Unit, Chinese University of Hong Kong, Trauma \& Emergency Centre, Prince of Wales Hospital, Shatin, NT, Hong Kong SAR; jo.chungym@gmail.com

Accepted 11 September 2012 Published Online First 25 October 2012

\title{
The effectiveness of a specially designed shoulder chair for closed reduction of acute shoulder dislocation in the emergency department: a randomised control trial
}

\author{
Josephine Yuen Man Chung, ${ }^{1,2}$ Chi Hung Cheng, ${ }^{1,2}$ Colin A Graham, ${ }^{1,2}$ \\ Timothy H Rainer ${ }^{1,2}$
}

\begin{abstract}
Study objective The objective of this study was to demonstrate the effectiveness of a specially designed chair for closed reduction of acute shoulder dislocations.

Method This was a prospective, non-blinded randomised controlled trial conducted in a university affiliated emergency department (ED). The inclusion criteria were (1) age $\geq 18$ years; (2) anterior or posterior shoulder dislocation without fracture of the surgical neck of the humerus; (3) patient who is able to communicate and cooperate. Participants were randomly assigned using a computer generated random number sequence into one of two groups - either the traditional practice group or Oxford chair group. Administration of intravenous sedation was only permitted in the traditional practice group due to the concerns of sedation use in the sitting position while unsupported on the chair. The primary outcome measure was length of ED stay. The secondary outcome measures were length of time for the procedure, successful reduction rate, levels of pain experienced by patients in different time periods before and after the reduction.
\end{abstract}

Result Sixty eligible patients were recruited, 30 in each group. The median lengths of stay in the ED in Oxford chair group $(n=30)$ and traditional method group $(n=30)$ were152 min and 173 min respectively $(p=0.183)$. The median procedure time was 3 min for the Oxford chair group compared to $5 \mathrm{~min}$ in the traditional method group $(p=0.179)$. The success rate for the Oxford chair method was $77 \%$ (23/30). There were no statistically or clinically significant differences of pain score at any point. Conclusions The chair method had a $77 \%$ success rate in reducing acute shoulder dislocations without sedation. There was no difference in pain level experienced by patients between the chair method and the traditional method. Patient factors, including patients who have had previous shoulder surgery and patients who have fracture dislocations, contribute to the reduced efficacy of the chair method. It remains possible that the chair method may reduce patient length of stay in the $E D$ in uncomplicated patients.

\section{INTRODUCTION}

Acute shoulder dislocation is a common problem which frequently leads patients to seek medical treatment in emergency departments (ED). Several methods exist for reducing shoulder dislocations. Some methods require the patient to lie supine, for example, the traction-countertraction technique, the Kocher procedure, the Hippocratic manoeuver, ${ }^{1}$ external rotation ${ }^{2}$ and the Milch-Cooper method. ${ }^{3}$ Some methods require the prone or sitting position, for example, scapular manipulation ${ }^{4}$ and the Stimson technique. ${ }^{1}$ These various methods had been classified into four categories: traction, leverage, scapular manipulation and combined manoeuvers. ${ }^{5}$

No single method has been shown to be superior to another and some techniques require considerable force and cause patient discomfort during the procedure. In practice, the chosen method used for shoulder reduction is highly dependent on the judgment of the treating clinician. Traditionally, analgesia or sedation is almost routinely required during the reduction procedure. Commonly recognised complications of sedating agents include airway obstruction, cardiorespiratory depression or instability, paradoxical or emergence reactions and vomiting/aspiration. Less commonly prolonged sedation, motor imbalance, agitation, renal impairment and allergy may occur. ${ }^{6}$ Patients should have close monitoring during the procedure and stay in the ED for observation after the procedure. ${ }^{7}$

Shoulder reduction without anaesthesia has been discussed by some authors, ${ }^{8-10}$ and several studies have subsequently attempted to demonstrate that a painless procedure could be achieved without any premedication. ${ }^{3}{ }^{11-15}$ A randomised prospective study reported by Fares et al ${ }^{15}$ showed that a new method of shoulder reduction had a higher success rate and less pain compared to the Hippocratic and Kocher methods. Baykal et al ${ }^{14}$ also showed a high success rate in shoulder reduction using a scapular manipulation technique without sedation. McNamara's study documented the level of pain experienced by patients during reduction without medication. Almost two-thirds of patients reported mild or no pain; the rest reported moderate to severe pain. ${ }^{12}$ However, few rigorous studies have been done to compare reduction methods without sedation with traditional practice.

Patients' comfort and relaxation are known to be key factors for successful shoulder reduction. ${ }^{16}$ The present study attempts to introduce an alternative shoulder reduction method using a specially designed chair (Oxford chair) to enhance patients' comfort and maximise relaxation during the procedure. Only one retrospective comparative study on the same method has been done by Smith, ${ }^{17}$ with a success rate of $62 \%$. No patient treated 




Photo: Specially designed shoulder reduction chair

with the Oxford chair required sedation and no complications were reported with the method. However, the levels of pain felt by patients were not documented in the study. Although, Smith's study ${ }^{17}$ attempted to compare the Oxford chair method with traditional methods, the comparison was not ideal as there was an imbalance between both groups in a retrospective study design.

\section{METHODS}

\section{Study design and setting}

This study was a prospective, non-blinded parallel group randomised controlled trial using a specially designed shoulder reduction chair (see photo) versus traditional shoulder reduction method to reduce acute shoulder dislocations in the ED. Each individual participant was randomly assigned into one of two parallel groups. Efforts were made to control for the extraneous variables of type of shoulder dislocation, amount and type of analgesic and sedation, and the reduction technique. This trial was registered and has the International Standard Randomised Controlled Trial Number (ISRCTN) 49254586.

The study was undertaken from November 2009 to August 2010 in the ED and trauma centre of Prince of Wales Hospital in the New Territories East Cluster of Hong Kong. The ED deals with around 150000 new patient attendances per annum and the hospital has 1400 beds. Ethical approval was obtained from the Joint CUHK-NTEC Clinical Research Ethics Committee. Subjects were provided essential information for informed consent and signed a consent form. The investigator explained the nature, purpose and the potential risk of the study to the subjects. Subjects were informed that they had any right to withdraw from the study without affecting the quality of care they received. The study was performed in accordance with the Declaration of Helsinki and in accordance with accepted international standards of good clinical practice in research.

\section{Population}

Patients aged 18 years or above with a diagnosis of anterior or posterior glenohumeral shoulder dislocation were invited to participate in the study. The inclusion criteria consisted of: (1) $\geq 18$ years; (2) anterior or posterior shoulder dislocation without fracture of the surgical neck of the humerus; (3) patient who was able to communicate and cooperate; (4) height $150 \mathrm{~cm}$ or above. The exclusion criteria were: (1) acute psychiatric disease; (2) inability to complete assessment of pain for example, dementia; (3) contraindications to any study medication.

\section{Study protocol}

Participants were randomly assigned to one of two groups by a computer generated random number sequence. This was completed prior to initiation of data collection and the allocations were sealed in opaque envelopes ready for consecutive patient use. Patients who met the inclusion criteria and consent to participate in the study were allocated a sealed opaque envelope with the technique for reduction inside. The sealed opaque envelopes were only opened to confirm the reduction technique after establishing the diagnosis and performing a clinical examination of the patient to detect possible neurovascular deficiencies.

In the traditional practice group, because currently no single shoulder reduction method has been proved to be superior to other methods, emergency clinicians could use any recognised method for closed reduction according to their preference and clinical judgment. In an attempt to control the extraneous variable of reduction technique, Kocher's manoeuver was suggested as a primary reduction technique. Physicians were asked to follow the pre-established study analgesic and sedation pathway. Oral Paracetamol $(1 \mathrm{~g})$ could be given to patients in both groups and patients in both groups were allowed rescue analgesia of intravenous Morphine $2 \mathrm{mg}$ (increments repeated as required) on request. In addition, for the traditional practice group, intravenous Midazolam $2 \mathrm{mg}$ would be used before performing the reduction. Physicians could titrate a further $1 \mathrm{mg}$ every $2-3 \mathrm{~min}$ to improve conditions to allow a satisfactory level of sedation for reduction.

In the shoulder chair group, shoulder reduction was performed by emergency physicians or emergency nurse practitioners. For patients in the shoulder chair group, sedation was not administered due to concerns about use of sedation in unsupported patients in the sitting position, without the security of a patient trolley. A specially designed shoulder chair with same measurements as the original one in the ED of John Radcliffe Hospital, Oxford, UK, was made. Two emergency nurse practitioners who were trained in the use of this chair provided training sessions to all emergency physicians in the study setting. A video clip demonstration of the technique was used as training material and hands on practice was included.

The front rest of the chair is positioned at an angle of about $45^{\circ}$, patients were instructed to sit on the chair with the anterior chest wall laying prone on the front rest. The affected arm rested with the axilla placed over the top of the front rest. The operator then applied relaxation techniques, such as used soft voice to instruct patient doing deep breathings and dimming the room light, encouraging relaxation of the patient. When patients became more relaxed he grasped the patient's forearm and applied longitudinal traction in conjunction with gravity. The operator could apply gentle external rotation to the shoulder in order to facilitate shoulder joint reduction.

In the chair group, when a patients' shoulder failed to be reduced with the chair method by three attempts or patients requested sedation for reduction, they were then treated using traditional practice with sedation. Patients who had a failed reduction using both methods were admitted to the orthopaedic service for reduction under formal general anaesthesia.

Data was recorded using a data collection form which consisted of three parts. The first part documented patient characteristics, such as age, gender, history of direct trauma, type of shoulder dislocation and recurrence of dislocation, etc. 
Figure 1 CONSORT Flow Diagram describing progress of patients through randomised trial. Access the article online to view this figure in colour.

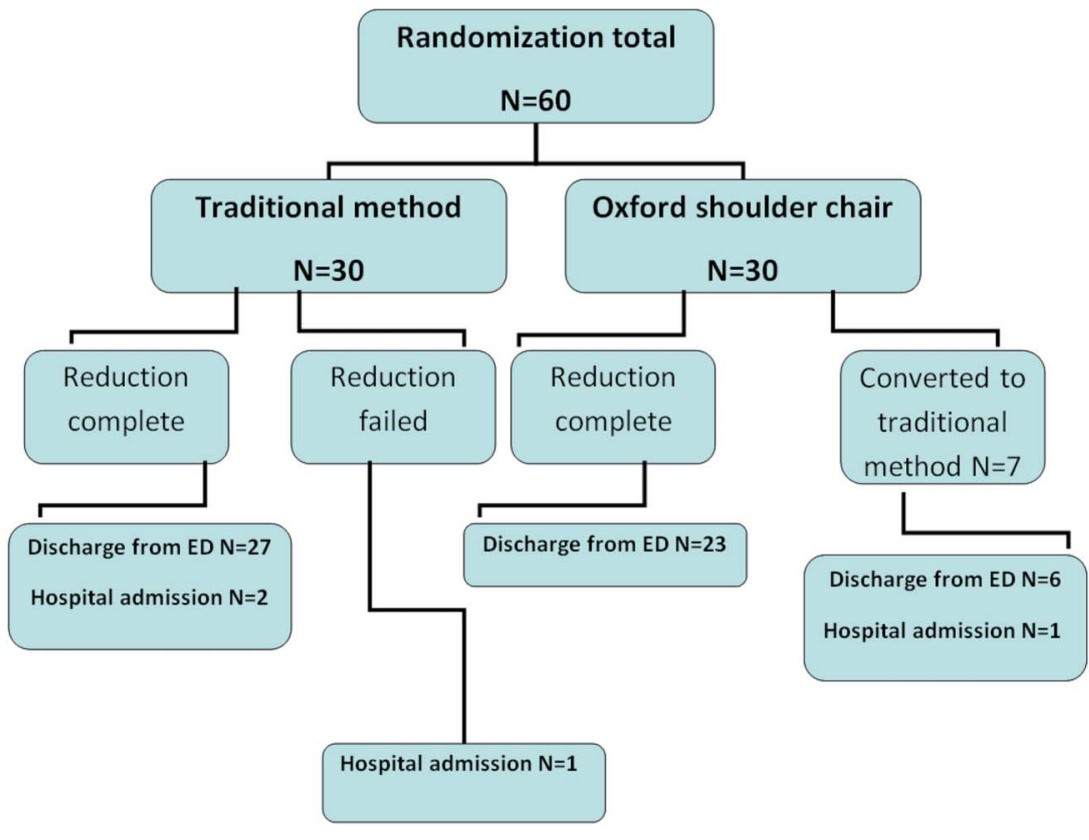

The second part documented data during the procedure, such as procedure time, initial and final reduction method, reduction outcome and patient disposition, etc. The third part documented the level of pain felt by patient at triage, before, during, $10 \mathrm{~min}$ after the procedure and on disposal. The $100 \mathrm{~mm}$ visual analogue scale was adopted for the measurement of pain. Data collection occurred $24 \mathrm{~h}$ per day.

\section{Key outcome measures}

The primary outcome measure for this study was the length of patient stay in the ED. The secondary outcome measures were length of the procedure; successful reduction rate; levels of pain experienced by patients in different time periods before and after the reduction.

\section{Data analysis}

Between 1 January 2008 and 30 April 2008, the mean length of ED stay for patients with acute shoulder dislocation (with shoulder reduction performed in the usual manner) was $148 \mathrm{~min}(\mathrm{n}=9$, SD 82). Based on this preliminary data, the sample size required to show a decrease in mean time from $148 \mathrm{~min}$ to $90 \mathrm{~min}$ with power of 0.8 (type II error of 0.2 ) and a type I error of 0.05 would be 30 patients in each of two groups.

Data were analysed on an intention-to-treat basis. Data were entered and analysed using the SPSS/PC V.16.0 software package. All raw data entered into the computer were checked for errors. Patient characteristics data were analysed with frequencies, per cents, means, SD, and ranges and presented in tables. Means, SD, median and ranges were calculated on the length of the procedure and length of patient stay. The t-test and Fisher's Exact test were used to compare statistical differences of two groups. The success rate of the two methods was presented as percentages.

\section{RESULTS}

During the data collection period from November 2009 to July 2010, 60 eligible patients presented to the ED were recruited to the study. All 60 patients consenting to participate in the study were randomly allocated to one of two study groups. The flow of participants through the trial is shown in figure 1 . There were 30 patients in each study group. In the Oxford chair group, reduction failed in seven patients who then went on to have a successful reduction using the traditional method. Only one patient had a failed reduction in the traditional method group and was admitted to the orthopaedic service. The two treatment groups had similar baseline characteristics (table 1).

The primary outcome measure of the median length of stay in ED was $152 \mathrm{~min}$ (95\% CI 82 to 235) in the Oxford chair group compared to $173 \mathrm{~min}(95 \%$ CI 126 to 246$)$ in the traditional method group, $21 \mathrm{~min}$ shorter for the chair group (figure 2). For the secondary outcomes, the total median procedure times were similar in both groups (figure 3 ). The success rate was 97\% (29/30) for the traditional method group and $77 \%$ (23/30) for the Oxford chair group (table 1). There were no differences observed in mean pain score at baseline, $30 \mathrm{~min}$

Table 1 Baseline characteristic between two randomisation groups

\begin{tabular}{lccc}
\hline & $\begin{array}{c}\text { Traditional } \\
\text { method } \\
\mathbf{N = 3 0}\end{array}$ & $\begin{array}{l}\text { Oxford shoulder } \\
\text { chair } \\
\mathbf{N = 3 0}\end{array}$ & $\begin{array}{l}\text { p } \\
\text { Value }\end{array}$ \\
\hline Age, mean (SD) & $50.1(23.15)$ & $43.7(22.51)$ & 0.48 \\
Female, number (\%) & $17(57)$ & $10(33)$ & 0.69 \\
Male, number (\%) & $13(43)$ & $20(67)$ & 0.69 \\
Recurrent dislocation number (\%) & $14(47)$ & $17(57)$ & 0.3 \\
History of previous surgery & $0(0)$ & $2(7)$ & 0.49 \\
number (\%) & $0(0)$ & $0(0)$ & 1 \\
History of shoulder fracture & & & \\
number (\%) & $27(90 \%)$ & $28(94 \%)$ & 0.64 \\
Neurological test before reduction & $3(10 \%)$ & $2(7 \%)$ & 0.64 \\
$\quad$ Intact, number (\%) & & & \\
$\quad$ Nerve deficit, number (\%) & $21(70)$ & $20(67)$ & 0.78 \\
Traumatic status & $9(30)$ & $10(33)$ & 0.78 \\
$\quad \begin{array}{l}\text { Traumatic, number (\%) } \\
\text { Non-traumatic, number (\%) }\end{array}$ & $157.3(98.13)$ & $177.7(197.7)$ & 0.17 \\
$\quad$ Time from dislocation to & & & 0.023 \\
reduction Mean (SD) & & & \\
Initial method outcome & & & \\
Successful, number (\%) & & & \\
\hline
\end{tabular}


Box Plot

Grouping Variable(s): Rand Group

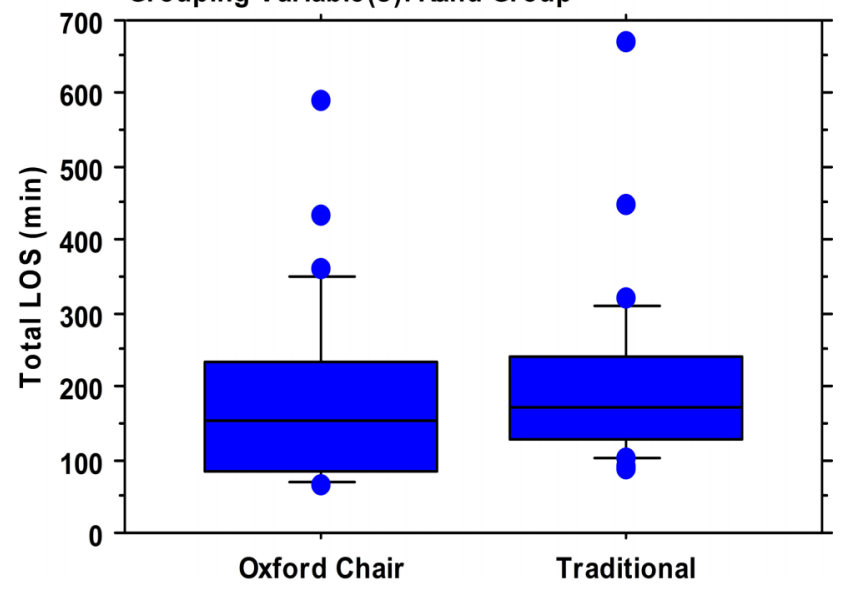

Figure 2 Box plot shows total EDLOS Emergency Department Length of Stay (minutes) between two Randomisation methods. Access the article online to view this figure in colour.

after oral analgesic, during procedure, $10 \mathrm{~min}$ after the procedure and on discharge (figure 4). All dislocations in this study were anterior dislocations. There were no adverse events reported, and specifically no adverse events due to sedation.

When groups were compared on a per-protocol basis, there was a significantly shorter LOS Length of Stay for the Oxford chair group compared to traditional reduction group (median $125 \mathrm{~min},(95 \% \mathrm{CI} 78$ to 180$), \mathrm{n}=23$ vs median $190 \mathrm{~min}$ (95\% CI 128.5 to 270.5$), n=37 ; p<0.001$, Mann-Whitney $\mathrm{U}$ test) (figure 5 ). There was also a significantly shorter procedure time for the Oxford chair group compared to the traditional reduction group (median $2 \min (95 \% \mathrm{CI} 1$ to 4$), \mathrm{n}=23$ vs median $6 \min (95 \% \mathrm{CI} 3$ to 9), $n=37 ; p<0.001$, Mann-Whitney U test) (figure 6).

When the 23 first time reductions using the chair were compared to the 29 patients who had a successful first time traditional reduction, there was significant different in LOS and procedure time between these groups (table 2).

\section{DISCUSSION}

In the present trial, the success rate of shoulder reduction using Oxford chair was $77 \%$ which is higher than a recent published retrospective study of the same technique (success rate $62 \%) .{ }^{17}$

Box Plot

Grouping Variable(s): Rand Group



Figure 3 Box plot shows total procedure time (minutes) between two Randomisation methods. Access the article online to view this figure in colour.

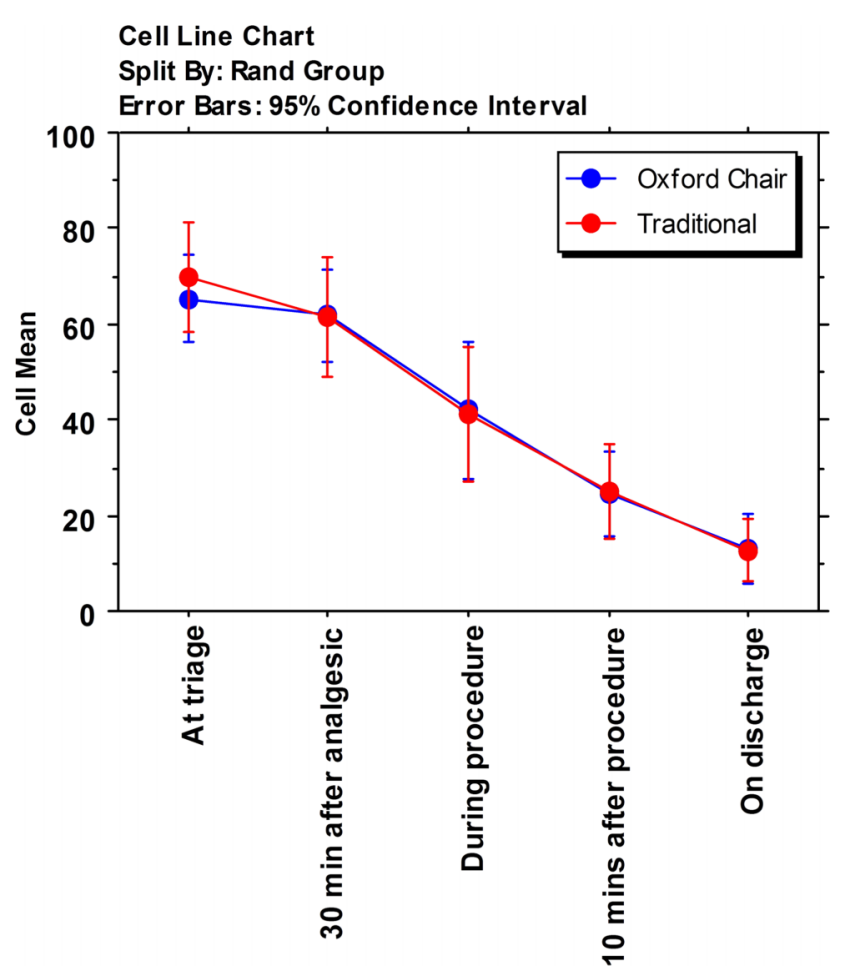

Figure 4 Cell line chart shows pain score (mean) difference with $95 \% \mathrm{Cl}$ between two random methods $(\mathrm{N}=60)$. Access the article online to view this figure in colour.

Currently, no single shoulder reduction method has a $100 \%$ success rate and nor is any technique ideal in every situation of shoulder dislocation. The reported success rate using different methods in studies ranges from $70 \%$ to $96 \% .{ }^{18}$

Overcoming shoulder muscle spasm is paramount to successful shoulder reduction. To achieve adequate patient relaxation traditionally, intravenous sedation has almost always been felt to be necessary. A survey in Ireland attempted to understand the general management of shoulder dislocation among emergency and orthopaedic specialists, the majority of participants reported that they routinely use sedation or analgesia for the reduction. ${ }^{19}$ Complications of sedation include residual sedative effect, respiratory complications, cardiovascular complications, permanent brain damage and death. ${ }^{20}$ Unnecessary sedation

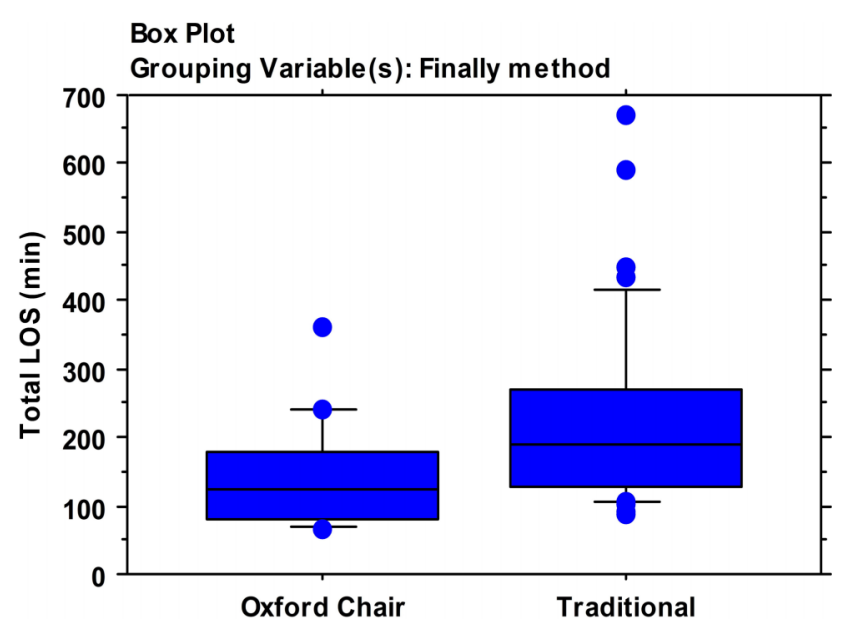

Figure 5 Box plot shows total EDLOS (minutes) between two Final methods. Access the article online to view this figure in colour. 
Box Plot

Grouping Variable(s): Finally method

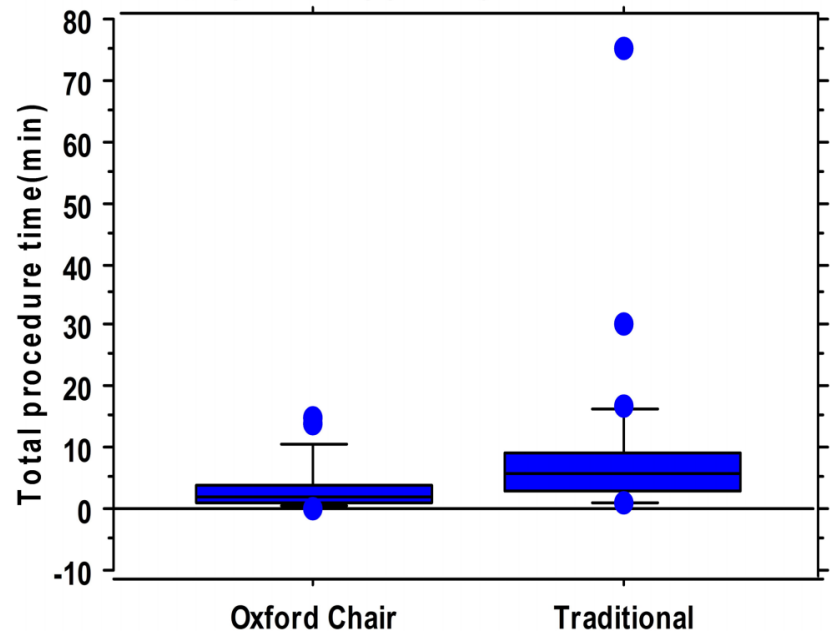

Figure 6 Box plot shows total procedure time (minutes) between two Final methods. Access the article online to view this figure in colour.

should be avoided to reduce the chances of potential complications wherever possible. Furthermore, the benefits of reduction without sedation can save staff time in the ED and facilitate rapid patient disposition. ${ }^{21}$

Reduction without sedation has been debated in the literature for over a decade. Numerous studies have demonstrated different techniques or manoeuvers for shoulder reduction without sedation and shown satisfactory success rates, with few patients encountering complications. ${ }^{14} 152223$ Instead of using forceful reduction techniques, the similarity of these skills is the adoption of gentle approaches, such as prone position, hanging weight or scapular manipulation, facilitating patient relaxation and reducing shoulder muscle spasm. Moreover, single operator is usually sufficient to complete the reduction with these methods, such as Stimson and scapular manipulation. However, a disadvantage of the Stimson method is that it requires the patient to be in the prone position with the weight attached to the arm for 20-30 min to achieve the reduction. This is often difficult for a busy ED allocating staff to monitor the patient. In this trial, median procedure time was $3 \mathrm{~min}$ and a single operator is sufficient to complete the procedure.

\section{Limitations}

Our primary outcome measure may be prone to bias in a nonblinded randomised design. However, it was not possible to blind the intervention and the randomised design of the study should minimise any differences between the groups. Furthermore, the two important patient-centred variables were

Table 2 Outcome between two methods in successful reductions

\begin{tabular}{lccc}
\hline Successful method groups & $\begin{array}{c}\text { Traditional method } \\
\mathbf{N}=\mathbf{2 9}\end{array}$ & $\begin{array}{c}\mathbf{0 x f o r d} \text { shoulder chair } \\
\mathbf{N}=\mathbf{2 3}\end{array}$ & p Value* \\
\hline Reduction time, minutest & $5(3-8)$ & $2(1-4)$ & 0.008 \\
ED Length of Stay, minutest & $180(125.5-253)$ & $125(78-180)$ & 0.025 \\
\hline
\end{tabular}

*Mann-Whitney.

tData presented as median (25th to 75th quartiles).

$E D$, emergency department. sedation and time in the ED, and the primary outcome measure was chosen to reflect that.

To our knowledge, this is the second study on the effectiveness of the Oxford chair for shoulder reduction; further multicentre studies should be able to confirm these outcomes. Based on the of characteristics of the seven patients who failed using the chair method, particular patients who have a history of shoulder problems such as previous surgery and fractures may have increased difficulty for shoulder reduction. We suggest that in future studies, these patients should be excluded and studies should focus on the potential benefits to those presenting with simple first time dislocations.

\section{CONCLUSION}

To conclude, the chair was successful in reducing dislocated shoulders in over three quarters of cases assigned for the method without sedation, and was associated with reduced ED length of stay in these cases. The chair method did not demonstrate as high an overall success rate as the traditional method of using sedation for reduction. Factors such as patients who were unable to relax, those who have had previous surgery on the shoulder, and those who have fracture dislocations, all contribute to decreasing the efficacy of reduction using the chair method. These situations notwithstanding, we suggest that the shoulder chair method is a safe and convenient method for reduction in patients who present with simple shoulder dislocations which are uncomplicated by fractures and previous shoulder surgery. Future studies focusing on patients presenting with simple dislocations only may prove to be worthwhile.

Acknowledgements The Hong Kong College of Emergency Medicine Research Grant.

Contributors THR is guarantor of the paper; he has overseen the entire planning, execution, and analysis of the study. JYMC had the idea for the study and obtained the ethical approval and grant for the study. JYMC and CHC participated in staff training in the application of the Oxford chair technique, recruitment of participants and data collection for the study. CAG and JYMC prepared the statistical analysis. CAG provided advice on the study methods and manuscript writing for the study. JYMC wrote the first draft of the paper and prepared the manuscript, and all authors have contributed to the final version. JYMC takes responsibility for the paper as a whole.

Funding The study has been conducted with the support of a Hong Kong College of Emergency Medicine Research Grant.

Competing interests None.

Provenance and peer reviewed Not commissioned; externally peer reviewed.

Presentation and award The primary data of the work described in the manuscript has been formally presented at the International Conference of Emergency Medicine in Singapore in 2010 and won a best oral presentation in the free paper session.

\section{REFERENCES}

1. McRae R, Esser M. Practical fracture treatment. 4th edn. London: Churchill Livingstone, 2004.

2. Danzl DF, Vicario SJ, Gleis GL, et al. Closed reduction of anterior subcoracoid shoulder dislocation. Evaluation of an external rotation method. Orthop Rev 1986;15:311-5.

3. O' Connor DR, Schwarze D, Fragomen AT, et al. Painless reduction of acute anterior shoulder dislocation without anesthesia. Orthop 2006;29:528-32.

4. Kothari RU, Dronen SC. Prospective evaluation of the scapular manipulation technique in reducing anterior shoulder dislocations. Ann Emerg Med 1992;21:1349-52.

5. Riebel GD, McCabe JB. Anterior Shoulder dislocation: a review of reduction techniques. Am J Emerg Med 1991;9:180-8.

6. Miller MA, Levy P, Patel MM. Procedural sedation and analgesia in the emergency department: what are the risks? Emerg Med Clin North Am 2005;23:551-72.

7. Admas ST, Woods $\mathrm{C}$, Lyall $\mathrm{H}$, et al. Standards of practice in UK emergency departments before, during and after conscious sedation. Emerg Med J 2008;25:728-31. 
8. Doyle W, Ragar T. Use of the scapular manipulation method to reduce an anterior shoulder dislocation in the supine position. Ann Emerg Med 1996;27:92-4.

9. Kolb J, Krupnick J. Shoulder reduction without anesthesia. Ann Emerg Med 1996;28:581-2.

10. Garvanos C. Modification and improvements of the Milch technique for the reduction of anterior dislocation of the shoulder without premedication. J Trauma 1992;32:801-3.

11. Dimitri C, Hassan S, Andre L. Anteroinferior shoulder dislocation: an auto-reduction method without analgesia. J Orthop Trauma 1997;11:399-404.

12. McNamara RM. Reduction of anterior shoulder dislocation by scapular manipulation. Ann Emerg Med 1993;22:1140-4.

13. Kuah DEK. An alternative slump reduction technique of anterior shoulder dislocation: A 3-years prospective study. Clin J Sport Med 2000;10:158-61.

14. Baykal B, Sener S, Turkan H. Scapular manipulation technique for reduction of traumatic anterior shoulder dislocations: experience of an accident and emergency department. Emerg Med J 2005;22:336-8.

15. Fares ES, Eustathios I, Kenanidis KA, et al. Reduction of acute anterior dislocations: a prospective randomized study comparing a new technique with the Hippocratic and Kocher methods. J Bone Joint Surg 2009;91:2775-82.
16. Canales Cortes V, Garcia-Dihinx Checa L, Rodriguez Vela J. Reduction of acute anterior dislocation of the shoulder without anaesthesia in the position of maximum muscular relaxation. Int Orthop 1989:13:259-62.

17. Smith SL. An investigation comparing the Oxford Chair Technique with the traditional methods of glenohumeral dislocation reduction currently implemented Int Emerg Nurs 2009;17:38-46.

18. Roberts JR, Hedges JR. Clinical procedures in emergency medicine. 5th edn, USA: Saunders, 2010

19. Leonard M, Kiely P. Reduction of anterior shoulder dislocations: a basic treatment guideline based on clinical practice. Eur J Orthop Surg Traumatol 2007;17:561-5.

20. Hesion PM, Joshi GP. Sedation: Not quite that simple. Anesthesiol Clinician 2010;28:281-94.

21. Descamps MJL, Gwilym S, Weldon D, et al. Prospective audit of emergency department transit times associated with entonox analgesia for reduction of the acute, traumatic dislocated shoulder. Accid Emerg Nurs 2007;15:223-7.

22. Marinelli M, Palma L. The external rotation method for reduction of acute shoulder dislocations. J Orthopaed Traumatol 2009;10:17-20.

23. Ahmed SMY, Singh J, Nicol M. A stepped care of approach to reduction of anterior shoulder dislocation in the prone position. Surgeon 2007;5:363-67. 